TRANSACTIONS OF THE

AMERICAN MATHEMATICAL SOCIETY

Volume 351, Number 1, January 1999, Pages 353-362

S 0002-9947(99)02192-3

\title{
SCRAMBLED SETS OF CONTINUOUS MAPS OF 1-DIMENSIONAL POLYHEDRA
}

\author{
JIEHUA MAI
}

\begin{abstract}
Let $K$ be a 1-dimensional simplicial complex in $R^{3}$ without isolated vertexes, $X=|K|$ be the polyhedron of $K$ with the metric $d_{K}$ induced by $K$, and $f: X \rightarrow X$ be a continuous map. In this paper we prove that if $K$ is finite, then the interior of every scrambled set of $f$ in $X$ is empty. We also show that if $K$ is an infinite complex, then there exist continuous maps from $X$ to itself having scrambled sets with nonempty interiors, and if $X=R$ or $R_{+}$, then there exist $C^{\infty}$ maps of $X$ with the whole space $X$ being a scrambled set.
\end{abstract}

\section{INTRODUCTION}

Chaotic behavior is a manifestation of the complexity of nonlinear dynamical systems. There are some distinct definitions given by different authors. The following definition of chaos mainly stems from Li and Yorke [11].

Definition 1.1. Let $(X, d)$ be a metric space, and $f: X \rightarrow X$ be a continuous map. A subset $S$ of $X$ containing at least two points is called a scrambled set of $f$ if for any $x, y \in S$ with $x \neq y$,

$$
\limsup _{n \rightarrow \infty} d\left(f^{n}(x), f^{n}(y)\right)>0,
$$

and

$$
\liminf _{n \rightarrow \infty} d\left(f^{n}(x), f^{n}(y)\right)=0 .
$$

$f$ is said to be chaotic (in the sense of Li and Yorke) if $f$ has an uncountable scrambled set.

Remark 1.2. Let $P(f)$ denote the set of all periodic points of $f$. In Definition 1.1, we do not insist that

$$
\limsup _{n \rightarrow \infty} d\left(f^{n}(x), f^{n}(p)\right)>0
$$

holds for any $x \in S$ and any $p \in P(f)$ because condition (1.3) is not important. In fact, if (1.1) and (1.2) hold for any $x, y \in S$ with $x \neq y$, then the set

$$
\left\{x \in S: \limsup _{n \rightarrow \infty} d\left(f^{n}(x), f^{n}(p)\right)=0 \text { for some } p \in P(f)\right\}
$$

Received by the editors January 30, 1997.

1991 Mathematics Subject Classification. Primary 58F13; Secondary 58F08, 54H20.

Key words and phrases. Chaos, 1-dimensional polyhedron, scrambled set, totally chaotic map. This work supported by National Natural Science Foundation of China. 
contains at most one point. Also we do not insist that $S \cap P(f)=\emptyset$ because if (1.2) holds, then $S \cap P(f)$ also contains at most one point (see (ii) of Lemma 2.1 below).

Let $I$ be a compact interval. For the case $X=I, \mathrm{Li}$ and Yorke in [11] first showed that if $f: I \rightarrow I$ has a periodic point of period 3 , then it is chaotic, i.e. "period three implies chaos". Kuchta and Smital in [9] indicated that if $f: I \rightarrow I$ has a two point scrambled set, then it has an uncountable scrambled set. In [5]-[8], [12], [13] and [15], scrambled sets of some maps were further discussed from the point of view of measure.

In this paper we will consider the case of $X$ being a 1-dimensional polyhedron. The tree is a particular kind of 1-dimensional polyhedron. In [1], [2], [3] and [10], the sets of periods of periodic orbits and the topological entropies of tree maps were discussed. Now we will study scrambled sets of continuous maps for general 1-dimensional polyhedra from the point of view of topology. Our main result is the following theorem.

Theorem A. Let $K$ be a finite 1-dimensional simplicial complex in $R^{3}$ without isolated vertexes, and let $X=|K|$ be the polyhedron of $K$. Suppose $f: X \rightarrow X$ is a continuous map. Then the interior of any scrambled set of $f$ in $X$ is empty.

In addition, we will show that if $K$ is an infinite 1-dimensional complex, then Theorem A is not true. Particularly, if $K$ is a triangulation of $R$ or $R_{+}$, i.e., if $X$ is the real line $R$ or the real half-line $R_{+}$, then there exist $C^{\infty}$ maps from $X$ to itself with the whole space $X$ being a scrambled set.

\section{Some Elementary Properties of SCRAmbled Sets}

Let $(X, d)$ be a metric space, and $f: X \rightarrow X$ be a continuous map. A point $x \in X$ is called an eventually periodic point of $f$ if there are integers $n>m \geq 0$ such that $f^{n}(x)=f^{m}(x)$. If $m=0$, i.e. $f^{n}(x)=x$, then $x$ is called a periodic point.

The following lemma will be useful, of which the proof is easy and is omitted.

Lemma 2.1. Let $S$ be a scrambled set of $f: X \rightarrow X$. Then

(i) $f \mid S$ is an injection.

(ii) There is at most one eventually periodic point of $f$ in $S$.

(iii) For any integer $n \geq 0, f^{n}(S)$ is also a scrambled set of $f$.

(iv) Let $S^{\prime} \subset X$ contain at least two points. If $f\left(S^{\prime}\right) \subset S$ and $f \mid S^{\prime}$ is injective, then $S^{\prime}$ is also a scrambled set of $f$.

(v) If $f$ is uniformly continuous, then $S$ is also a scrambled set of $f^{n}$ for any integer $n>0$.

Definition 2.2. Let $(X, d)$ and $\left(X^{\prime}, d^{\prime}\right)$ be two metric spaces, and $h: X \rightarrow X^{\prime}$ be a homeomorphism. $h$ is called a uniform homeomorphism if both $h$ and $h^{-1}$ are uniformly continuous. $X$ and $X^{\prime}$ are said to be uniformly homeomorphic if there exists a uniform homeomorphism $h: X \rightarrow X^{\prime}$.

Obviously, every homeomorphism between two compact metric spaces must be a uniform homeomorphism, and we have

Lemma 2.3. Let $h: X \rightarrow Y$ be a uniform homeomorphism, and $S \subset X, T=h(S)$. Suppose $f: X \rightarrow X$ is a continuous map, and $g=h \circ f \circ h^{-1}$. Then $T$ is a scrambled set of $g$ if and only if $S$ is a scrambled set of $f$. 


\section{Scrambled Sets of CONTINUOUS MAPS OF 1-DIMENSIONAL POLYHEDRA}

Let $K$ be a 1-dimensional simplicial complex in $R^{3}$. Every 0-dimensional simplex of $K$ is called a vertex, and every 1-dimensional simplex of $K$ is called an edge. Denote by $K_{0}$ the set of all vertexes of $K$. Let $X=|K|$ be the polyhedron of $K$ (see [4]). Define the metric $d_{K}$ on $X$ as follows:

(i) If points $x$ and $y$ lie on the same edge $E$, the two vertexes of $E$ are $u$ and $v$, and $x=r u+(1-r) v, y=s u+(1-s) v$ for some $r, s \in[0,1]$, then $d_{K}(x, y)=|r-s|$.

(ii) If there is a connected subcomplex $K^{\prime}$ of $K$ such that $\{x, y\} \subset\left|K^{\prime}\right|$, then

$$
\begin{gathered}
d_{K}(x, y)=\min \left\{\sum_{i=1}^{n} d_{K}\left(x_{i-1}, x_{i}\right):\left(x_{0}, x_{1}, \ldots, x_{n}\right)\right. \text { is a sequence of points } \\
\quad \text { in } X \text { with } x_{0}=x, x_{n}=y, \text { and } x_{i-1} \text { and } x_{i} \text { lying } \\
\text { on the same edge of } K \text { for } i=1, \ldots, n\} .
\end{gathered}
$$

(iii) If there is no connected subcomplex $K^{\prime}$ of $K$ such that $\{x, y\} \subset\left|K^{\prime}\right|$, then $d_{K}(x, y)=\infty$.

Remark 3.1. In order to avoid that the case $d_{K}(x, y)=\infty$ arises, we can give another metric $d_{K}^{\prime}$ on $X$ by

$$
d_{K}^{\prime}(x, y)= \begin{cases}\operatorname{arctg} d_{K}(x, y), & \text { if } d_{K}(x, y)<\infty \\ 1, & \text { if } d_{K}(x, y)=\infty\end{cases}
$$

However, it is easy to see that the identical map $i d:\left(X, d_{K}\right) \rightarrow\left(X, d_{K}^{\prime}\right)$ is a uniform homeomorphism. Thus, for convenience, we use $d_{K}$ rather than $d_{K}^{\prime}$.

Remark 3.2. If for any bounded subset $B$ of $R^{3}$, the number of the simplexes of $K$ intersecting $B$ is finite, then the topology on $X$ induced by $d_{K}$ coincides with that as subspace of the Euclidean space $R^{3}$.

Recall that an arc is a space homeomorphic to the unit interval $[0,1]$. Let $A \subset X$ be an arc. Denote by $\partial A$ the two endpoints of $A$, and write $\stackrel{\circ}{A}=A-\partial A$. Let $x$ and $y$ be two points on arc $A$. Denote by $A[x, y]$ the subarc of $A$ from $x$ to $y$. If $A[x, y]$ is a straight line segment, then it is simply written as $[x, y]$, and put $(x, y]=[x, y]-\{x\},(x, y)=(x, y]-\{y\}$. Let $u$ and $v$ be the two endpoints of $A$. We denote by $(A ; u, v)$ the directed arc $A$ from $u$ to $v$. In addition, we denote by $l(A)$ the length of arc $A$ under metric $d_{K}$.

Lemma 3.3. Let $(A ; u, v)$ be a directed arc on $X=|K|$. Suppose $\{u, v\} \cap K_{0}=\emptyset$. Then there is a unique sequence $\left(w_{0}, w_{1}, \cdots, w_{n}\right)$ of vertexes of $K$ with $n \geq 1$ satisfying the following four conditions:

(i) For $i=1,2, \cdots, n,\left[w_{i-1}, w_{i}\right]$ is an edge of $K$.

(ii) $u \in\left[w_{0}, w_{1}\right), v \in\left(w_{n-1}, w_{n}\right]$.

(iii) If $n=1$, then $u \in\left[w_{0}, v\right]$ and $A=[u, v]$. If $n>1$, then

$$
A=\left[u, w_{1}\right] \cup\left(\bigcup_{i=2}^{n-1}\left[w_{i-1}, w_{i}\right]\right) \cup\left[w_{n-1}, v\right] .
$$

(iv) $w_{i} \neq w_{j}$ for $1 \leq i<j \leq n-1$. 
Lemma 3.3 is evident. The sequence $\left(w_{0}, w_{1}, \cdots, w_{n}\right)$ in Lemma 3.3 will be called the carrier sequence of the directed $\operatorname{arc}(A ; u, v)$ and we write $\operatorname{CS}(A ; u, v)=$ $\left(w_{0}, w_{1}, \cdots, w_{n}\right)$.

Theorem A. Let $K$ be a finite 1-dimensional simplicial complex in $R^{3}$ without isolated vertexes, and let $X=|K|$ be the polyhedron of $K$. Suppose $f: X \rightarrow X$ is a continuous map. Then the interior of any scrambled set of $f$ in $X$ is empty.

Proof. If not, there is a scrambled set $S$ of $f$ having a nonempty interior in $X$. Then $S$ contains an arc $L$. By (ii) of Lemma 2.1, we may assume that $L$ contains no eventually periodic points of $f$. By (i) of Lemma 2.1, $f^{k}(L)$ is also an arc in $X(k=1,2, \cdots)$. Let the two endpoints of $L$ be $x^{\prime}$ and $y^{\prime}$. By Definition 1.1, we have $\limsup _{k \rightarrow \infty} d_{k}\left(f^{k}\left(x^{\prime}\right), f^{k}\left(y^{\prime}\right)\right)>0$. Thus $\sum_{k=0}^{\infty} l\left(f^{k}(L)\right)=\infty$. This implies that $f^{\mu}(L) \cap f^{m}(L) \neq \emptyset$ for some integers $\mu>m \geq 0$. Write $A^{\prime}=f^{m}(L)$. Take $u, v \in A^{\prime}$ such that $v=f^{\mu-m}(u)$ and $l\left(A^{\prime}[u, v]\right)$ achieves the minimum. Let $A=A^{\prime}[u, v]$, and $g=f^{\mu-m}$. By (iii) and (v) of Lemma 2.1 we know that $A \subset A^{\prime}$ is a scrambled set of $g$. Write $A_{k}=g^{k}(A)$ and $u_{k}=g^{k}(u)$ for $k=0,1, \cdots$. Then $v=g(u)=u_{1}$. It follows from (i) of Lemma 2.1 that $A_{k}$ is an arc and the two endpoints of $A_{k}$ are $u_{k}$ and $u_{k+1}$. Since $A \cap P(g)=\emptyset$, we have

$$
A_{i} \not \subset A_{j}, \quad \text { for any nonnegative integers } i \neq j \text {. }
$$

From (3.1) we get the following

Claim 1. Let $k \geq 0$. If there exist edges $E$ and $E^{\prime}$ of $K$ such that $A_{k} \subset E$ and $A_{k+1} \subset E^{\prime}$, then $A_{k} \cap A_{k+1}=\left\{u_{k+1}\right\}$, and $E=E^{\prime}$ if $u_{k+1} \notin K_{0}$.

Since $K_{0}$ is a finite set and $A$ contains no eventually periodic points of $g$, there is a $k_{0} \geq 0$ such that $u_{k} \notin K_{0}$ for all $k \geq k_{0}$. Noting $\lim _{\sup _{k \rightarrow \infty}} l\left(A_{k}\right)>0$, by (3.1) and Claim 1 we have

Claim 2. Write $Z_{0}=\left\{k: k \geq k_{0}+2\right.$, and $\left.A_{k} \cap K_{0} \neq \emptyset\right\}$. Then $Z_{0}$ is an infinite set.

By (iv) of Lemma 3.3, the number of carrier sequences of all directed $\operatorname{arcs}$ in $X$ is finite. Hence there exist integers $a$ and $b \in Z_{0}$ with $|a-b| \geq 3$ such that

$$
\begin{aligned}
\operatorname{CS}\left(A_{a} ; u_{a}, u_{a+1}\right) & =\operatorname{CS}\left(A_{b} ; u_{b}, u_{b+1}\right), \\
\mathrm{CS}\left(A_{a+1} ; u_{a+1}, u_{a+2}\right) & =\operatorname{CS}\left(A_{b+1} ; u_{b+1}, u_{b+2}\right) .
\end{aligned}
$$

Suppose the carrier sequence $\operatorname{CS}\left(A_{a} ; u_{a}, u_{a+1}\right)$ is $\left(v_{0}, v_{1}, \cdots, v_{n}\right)$. Then $n \geq 2$. By (3.2), we have $u_{a} \in\left(v_{0}, u_{b}\right)$ or $u_{b} \in\left(v_{0}, u_{a}\right)$. By the symmetry, we may assume that

$$
u_{a} \in\left(v_{0}, u_{b}\right)
$$

It follows from (3.4), (3.2) and (3.1) that $u_{b} \in\left(u_{a}, v_{1}\right)$, and $u_{a+1} \in\left(v_{n-1}, u_{b+1}\right)$, $u_{b+1} \in\left(u_{a+1}, v_{n}\right)$. We now claim

$$
g\left(\left[u_{a}, u_{b}\right]\right)=\left[u_{a+1}, u_{b+1}\right] .
$$

In fact, if (3.5) does not hold, then $g\left(\left[u_{a}, u_{b}\right]\right)$ is an arc in $X$ with endpoints $u_{a+1}$ and $u_{b+1}$ which does not intersect $\left(u_{a+1}, u_{b+1}\right)$. Noting $A_{a+1}=g\left(A_{a}\right)=g\left(\left[u_{a}, u_{b}\right]\right) \cup$ $g\left(A_{a}\left[u_{b}, u_{a+1}\right]\right)$ is an arc and $g \mid A_{a}$ is injective, we have

$$
g\left(A_{a}\left[u_{b}, u_{a+1}\right]\right) \subset\left[u_{b+1}, u_{a+1}\right), \text { and } u_{a+2} \in\left(u_{b+1}, u_{a+1}\right) .
$$




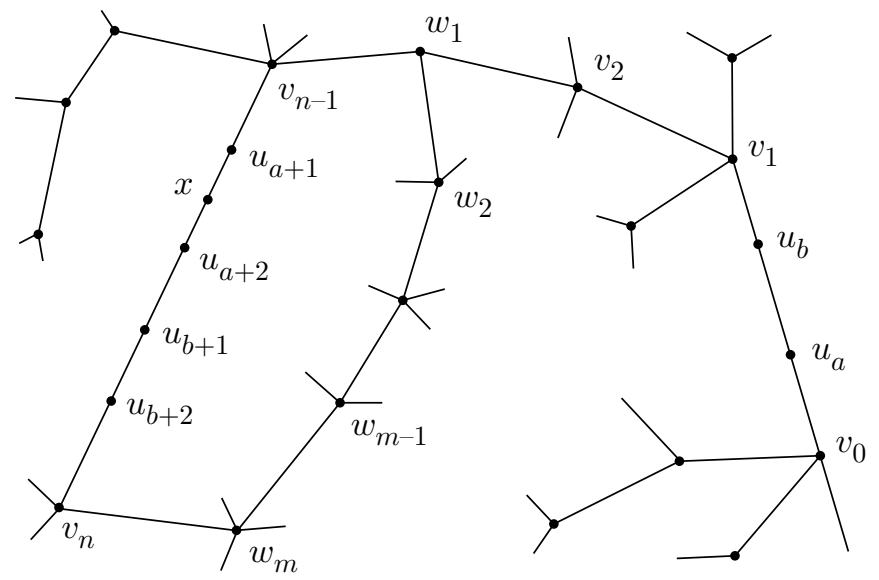

FiguRE 3.1

By (3.6) and (3.3) we get

$$
\begin{aligned}
\operatorname{CS}(g & \left.\left(\left[u_{a}, u_{b}\right]\right) ; u_{a+1}, u_{b+1}\right)=\operatorname{CS}\left(A_{a+1} ; u_{a+1}, u_{a+2}\right) \\
\quad & =\operatorname{CS}\left(A_{b+1} ; u_{b+1}, u_{b+2}\right)=\operatorname{CS}\left(g\left(\left[u_{a+1}, u_{b+1}\right]\right) ; u_{a+2}, u_{b+2}\right) \\
& =\left(v_{n}, v_{n-1}, w_{1}, \cdots, w_{m}, v_{n}, v_{n-1}\right), \quad \text { for some }\left\{w_{1}, \cdots, w_{m}\right\} \subset K_{0} .
\end{aligned}
$$

(see Fig. 3.1). This implies that there is a point $x \in\left(u_{a+1}, u_{b+1}\right)$ such that $g\left(\left[u_{a+1}, x\right]\right)=\left[u_{a+2}, v_{n-1}\right]$ and $g(x)=v_{n-1}$, and hence there is a fixed point $p$ of $g$ in $\left(u_{a+1}, x\right)$. However, $\left(u_{a+1}, x\right) \subset\left[u_{a+1}, u_{b+1}\right] \subset A_{b+1}$, which contains no fixed points of $g$. This leads to a contradiction. Thus (3.5) must hold.

From (3.5) and (i) of Lemma 2.1 it is easy to see that, for sufficiently small $\varepsilon>0, g\left(u_{b}+\varepsilon\left(v_{1}-u_{b}\right)\right) \in\left(u_{b+1}, v_{n}\right)-A_{b}$. Thus we have $u_{b}+\varepsilon\left(v_{1}-u_{b}\right) \notin A_{b-1}$ and hence

$$
\left[u_{a}, u_{b}\right] \subset A_{b-1} .
$$

There are two cases to consider:

Case 1. $b>a$. In this case, let $Q=\bigcup_{k=a}^{b} A_{k}$. By (3.5) and (3.7) we can easily verify that $g(Q)=Q$. Let $S^{1}=\left\{e^{2 \pi i t}: t \in R\right\}$ be the unit circle in the complex plane, and $d$ be the usual metric on $S^{1}$. Take a sequence $t_{a}<t_{a+1}<\cdots<t_{b}$ of real numbers such that $t_{b-1}<t_{a}+1<t_{b}<t_{a+1}+1$. Put $z_{k}=e^{2 \pi i t_{k}}$ for $k=a, a+1, \cdots, b$. Let $C_{k}=\left\{e^{2 \pi i t}: t_{k} \leq t \leq t_{k+1}\right\}$ for $k=a+1, \cdots, b-2, b-1$, and let $C_{b}=\left\{e^{2 \pi i t}: t_{b} \leq t \leq t_{a+1}+1\right\}$ (see Fig. 3.2). Then $C_{j}$ is an arc on $S^{1}(j=a+1, \cdots, b)$. For $k=a+1, \cdots, b-1$, choose a homeomorphism $h_{k}: C_{k} \rightarrow$ $A_{k}$ such that $h_{k}\left(z_{k}\right)=u_{k}, h_{k}\left(z_{k+1}\right)=u_{k+1}$, and $h_{b-1}\left(z_{a}\right)=u_{a}$. Choose again a homeomorphism $h_{b}: C_{b} \rightarrow A_{b}\left[u_{b}, u_{a+1}\right]$ such that $h_{b}\left(z_{b}\right)=u_{b}, h_{b}\left(z_{a+1}\right)=u_{a+1}$. Define a projection $h: S^{1} \rightarrow Q$ by $h \mid C_{k}=h_{k}$ for $k=a+1, \cdots, b$. Then $h$ is continuous, and $h\left(S^{1}\right)=Q$. Define $\varphi: S^{1} \rightarrow S^{1}$ by

$$
\begin{aligned}
& \varphi \mid C_{k}=h_{k+1}^{-1} \circ g \circ h_{k}, \quad \text { for } k=a+1, \cdots, b-2 ; \\
& \varphi\left|C_{b-1}\left[z_{b-1}, z_{a}\right]=h_{b}^{-1} \circ g \circ h_{b-1}\right| C_{b-1}\left[z_{b-1}, z_{a}\right] ; \\
& \varphi\left|C_{b-1}\left[z_{a}, z_{b}\right]=h_{a+1}^{-1} \circ g \circ h_{b-1}\right| C_{b-1}\left[z_{a}, z_{b}\right] ; \\
& \varphi \mid C_{b}=h_{a+1}^{-1} \circ g \circ h_{b} .
\end{aligned}
$$




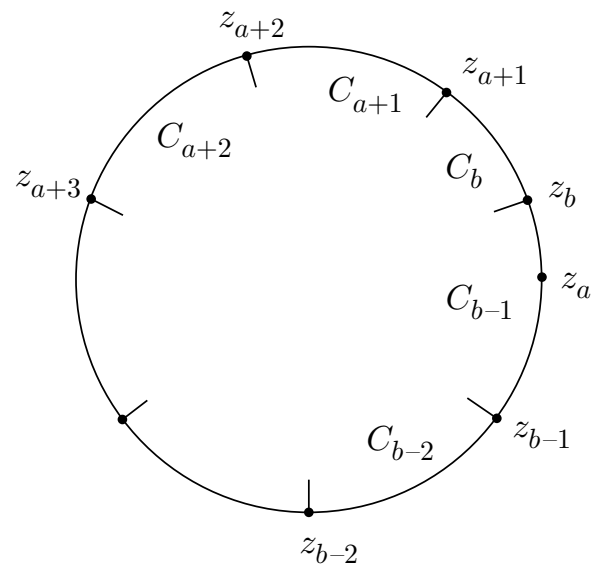

FiguRe 3.2

Then $\varphi$ is also continuous, and $h \circ \varphi=g \circ h$. We say that $\varphi$ is the lift of $g \mid Q$ relative to the projection $h$, or relative to the sequence $\left(h_{a+1}, h_{a+2}, \cdots, h_{b}\right)$ of homeomorphisms. Note that $\varphi: S^{1} \rightarrow S^{1}$ is both injective and surjective. Thus $\varphi$ is a homeomorphism.

$\varphi$ has no periodic points because $g \mid Q$ has no periodic points and $h \circ \varphi=g \circ h$. This implies that the rotation number of $\varphi$ is irrational. If $\varphi$ has wandering points, then we know (for example, see [14, Chap.1]) that the wandering set $W(\varphi)$ of $\varphi$ is an open set dense in $S^{1}$. Let $z$ and $w$ be two different points on the same connected component of $W(\varphi) \cap C_{a+1}$. Then $\lim _{k \rightarrow \infty} d\left(\varphi^{k}(z), \varphi^{k}(w)\right)=0$ (see [14]). Since $S^{1}$ is compact, $h: S^{1} \rightarrow Q$ is uniformly continuous. Thus

$$
\lim _{k \rightarrow \infty} d_{K}\left(g^{k}(h(z)), g^{k}(h(w))\right)=\lim _{k \rightarrow \infty} d_{K}\left(h\left(\varphi^{k}(z)\right), h\left(\varphi^{k}(w)\right)\right)=0 .
$$

This implies that the points $h(z)$ and $h(w)(\neq h(z))$ of $A_{a+1}$ can not lie in the same scrambled set of $g$. However, as indicated above, $A$ is a scrambled set of $g$, and hence $A_{a+1}=g^{a+1}(A)$ is also a scrambled set. This reduces to a contradiction.

Therefore, $\varphi$ has no wandering points. Thus $\varphi$ is topologically conjugate to an irrational rotation of $S^{1}$, i.e. there exist an irrational number $c$ and an orientation preserving homeomorphism $\eta: S^{1} \rightarrow S^{1}$ such that

$$
\eta^{-1} \varphi \eta\left(e^{2 \pi i t}\right)=e^{2 \pi i(t+c)}, \quad \text { for any } t \in R .
$$

Let $\psi=\eta^{-1} \varphi \eta: S^{1} \rightarrow S^{1}$, and $\xi=h \eta: S^{1} \rightarrow Q$. Then $\xi$ is a continuous surjection, and

$$
\xi \circ \psi=g \circ \xi
$$

For any given positive number $r$, if there exists $s \in R$ such that $\xi\left(e^{2 \pi i(s+r)}\right)=$ $\xi\left(e^{2 \pi i s}\right)$, then it follows from (3.8) and (3.9) that

$$
\begin{array}{r}
\xi\left(e^{2 \pi i(s+k c+r)}\right)=\xi \psi^{k}\left(e^{2 \pi i(s+r)}\right)=g^{k} \xi\left(e^{2 \pi i(s+r)}\right) \\
=g^{k} \xi\left(e^{2 \pi i s}\right)=\xi \psi^{k}\left(e^{2 \pi i s}\right)=\xi\left(e^{2 \pi i(s+k c)}\right)
\end{array}
$$


holds for all $k=0,1, \cdots$. Since the point set $\left\{e^{2 \pi i(s+k c)}: k=0,1, \cdots\right\}$ is dense in $S^{1}$, by (3.10) and the continuity of $\xi$ we have

$$
\xi\left(e^{2 \pi i(t+r)}\right)=\xi\left(e^{2 \pi i t}\right), \quad \text { for any } t \in R .
$$

Let $T=\left\{r: r \in(0,1]\right.$, and there exists $s=s(r) \in R$ such that $\xi\left(e^{2 \pi i(s+r)}\right)=$ $\left.\xi\left(e^{2 \pi i s}\right)\right\}$. Then $T$ is evidently a nonempty closed set in $(0,1]$. Let $r_{0}=\inf T$. Then $r_{0}>0$ since $\xi \mid \eta^{-1}\left(C_{a+1}\right)$ is a homeomorphism from $\eta^{-1}\left(C_{a+1}\right)$ to $A_{a+1} \subset Q$. It is easy to see that there is an integer $q \geq 1$ such that $r_{0}=1 / q$. By (3.11) and the definition of $r_{0}$ we know that, for any $t, t^{\prime} \in R, \xi\left(e^{2 \pi i t}\right)=\xi\left(e^{2 \pi i t^{\prime}}\right)$ if and only if $q\left(t^{\prime}-t\right)$ is an integer. Thus we can define $\zeta: S^{1} \rightarrow Q$ by

$$
\zeta\left(e^{2 \pi i t}\right)=\xi\left(e^{2 \pi i t / q}\right), \quad \text { for any } \quad t \in R .
$$

Obviously, this $\zeta$ is injective, surjective and continuous. Hence $\zeta$ is a homeomorphism. Define $\omega: S^{1} \rightarrow S^{1}$ by

$$
\omega\left(e^{2 \pi i t}\right)=e^{2 \pi i q t}, \quad \text { for any } t \in R .
$$

Then $\zeta \circ \omega=\xi$. Define $\Psi: S^{1} \rightarrow S^{1}$ by

$$
\Psi\left(e^{2 \pi i t}\right)=e^{2 \pi i(t+q c)}, \quad \text { for any } t \in R .
$$

Then $\Psi \circ \omega=\omega \circ \psi$. Therefore, we have the following commutative diagram.

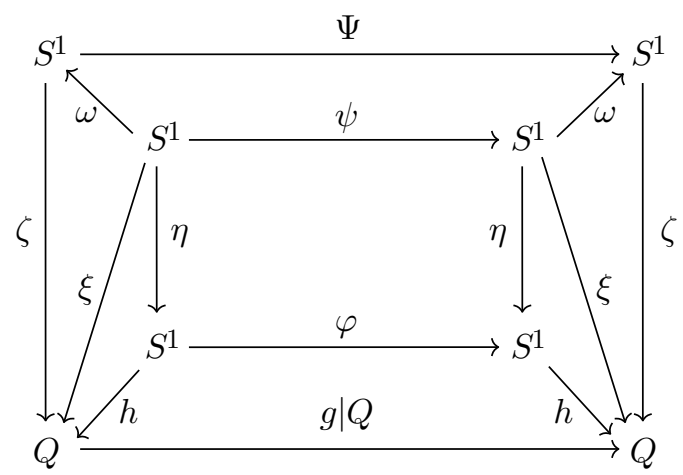

Thus $\zeta \Psi \omega=\zeta \omega \psi=\xi \psi=g \xi=g \zeta \omega$, and hence $\zeta \circ \Psi=g \circ \zeta$ because $\omega$ is a surjection. This implies that $g \mid Q$ and $\Psi$ are topologically conjugate. Since the irrational rotation $\Psi$ has no scrambled set, by Lemma $2.3, g \mid Q$ also has no scrambled set. However, as indicated above, $A_{a+1}(\subset Q)$ is a scrambled set of $g$. This is still a contradiction.

Case 2. $a>b$. Analogous to Case 1, Case 2 also leads to a contradiction.

Thus, the interior of any scrambled set of $f$ in $X$ must be empty. Theorem A is proven.

If complex $K$ is not finite, then Theorem $\mathrm{A}$ is not true. In fact, we have

Theorem 3.1. Let $K$ be an infinite 1-dimensional simplicial complex in $R^{3}$, and let $X=|K|$ be the polyhedron of $K$ with the metric $d_{K}$. Then there exists a continuous map $f: X \rightarrow X$ which has a scrambled set containing a nonempty interior in $X$.

Proof. If $K$ has isolated vertexes, take an edge $E$ of $K$, and let $f_{0}: E \rightarrow E$ be a continuous map which has an uncountable scrambled set $S_{0}$. Suppose the set 
of isolated vertexes of $K$ is $V$. Choose a continuous map $g: X \rightarrow E$ such that $g(x)=x$ for all $x \in E, g(V) \subset S_{0}$, and $g \mid V$ is injective. Let $f=f_{0} \circ g$, and let $S=S_{0} \cup V-g(V)$. Then $S$ is an uncountable scrambled set of $f$, the interior of $S$ in $X$ contains $V$, and is nonempty.

Now we assume that $K$ has no isolated vertexes. Then $K$ has infinitely many edges. Take countably infinitely many edges $E_{0}, E_{1}, E_{2}, \cdots$ of $K$ with $E_{i} \neq E_{j}$ for $i \neq j$ such that one of the following two conditions holds:

(C.1) If $K$ has infinitely many connected components, then for any $0 \leq i<$ $j<\infty, E_{i}$ and $E_{j}$ belong to different components of $K$.

(C.2) If $K$ has only finitely many connected components, then all of $E_{0}, E_{1}, E_{2}$, .. belong to the same component of $K$.

For $n=0,1,2, \cdots$, suppose $\partial E_{n}=\left\{v_{n}, w_{n}\right\}$. Let $x_{n}=\left(2 v_{n}+w_{n}\right) / 3, y_{n}=$ $\left(v_{n}+2 w_{n}\right) / 3$, and $A_{n}=\left[x_{n}, y_{n}\right]$. Denote by $J$ the open interval $(0,1)$. For any rational number $r \in J$, write $u_{n}(r)=(1-r) x_{n}+r y_{n}$. Put

$$
\begin{array}{r}
W=\{(a, b, r, s): a, b, r, s \text { are all rational numbers, } \\
\text { and } 0<a<b<1,0<r<s<1\} .
\end{array}
$$

Then $W$ is a countable set in $J^{4}\left(\subset R^{4}\right)$. Arrange all points in $W$ to be an infinite sequence. Assume the sequence is

$$
W=\left\{\left(a_{n}, b_{n}, r_{n}, s_{n}\right): n=0,1,2, \cdots\right\}
$$

For $n=1,2,3, \cdots$, choose a homeomorphism $g_{n}: A_{0} \rightarrow A_{n}$ such that $g_{n}\left(x_{0}\right)=$ $x_{n}, g_{n}\left(y_{0}\right)=y_{n}, g_{n}\left(u_{0}\left(a_{n}\right)\right)=u_{n}\left(r_{n}\right)$, and $g_{n}\left(u_{0}\left(b_{n}\right)\right)=u_{n}\left(s_{n}\right)$, and then define the homeomorphism $h_{n}: A_{n} \rightarrow A_{n+1}$ by $h_{n}=g_{n+1} \circ g_{n}^{-1}$. Let $h_{0}=g_{1}: A_{0} \rightarrow A_{1}$. Put $X_{0}=\bigcup_{n=0}^{\infty} A_{n}$. Define $f_{0}: X_{0} \rightarrow X_{0}$ by $f_{0} \mid A_{n}=h_{n}$ for $n=0,1,2, \cdots$. Then it is easy to see that $\stackrel{\circ}{A_{0}}$ is a scrambled set of $f_{0}$.

If condition (C.1) holds, suppose the connected component of $K$ containing $E_{n}$ is $K^{(n)}$, and $Y_{n}=\left|K^{(n)}\right|,(n=0,1,2, \cdots)$. Obviously, we can construct a continuous map $f: X \rightarrow X$ such that $f \mid X_{0}=f_{0}, f\left(Y_{n}\right)=E_{n+1}$ for $n=0,1,2, \cdots$, and $f(z)=z$ for any $z \in X-\bigcup_{n=0}^{\infty} Y_{n}$. Clearly, $\stackrel{\circ}{A}_{0}$ is still a scrambled set of $f$.

If condition (C.2) holds, put $X_{1}=X-\bigcup_{n=0}^{\infty} \stackrel{\circ}{E}_{n}$, and $X_{2}=X_{0} \cup X_{1}$. Define $f_{2}: X_{2} \rightarrow X_{2}$ by $f_{2} \mid X_{0}=f_{0}$, and $f_{2}(z)=z$ for any $z \in X_{1}$. Then $f_{2}$ is continuous.

Evidently, $f_{2}$ can be extented to be a continuous map $f: X \rightarrow X$, and $\stackrel{\circ}{A}_{0}$ is also a scrambled set of this $f$.

Note that $\stackrel{\circ}{A_{0}}$ is a nonempty open set in $X$. Theorem 3.1 is proven.

\section{Totally Chaotic Continuous maps of the ReAl Line}

In the proof of Theorem 3.1 we construct a continuous map $f: X \rightarrow X$, which has a scrambled set with a nonempty interior in $X$. But this $f$ is not a totally chaotic map defined as follows.

Definition 4.1. Let $(X, d)$ be a metric space. A continuous map $f: X \rightarrow X$ is called totally chaotic if the whole space $X$ is a scrambled set of $f$.

What metric space $X$ can admit a totally chaotic map? This is an interesting problem. In this section we will consider the real line $R$ and the real half-line $R_{+}=[0, \infty)$, which can be regarded as the polyhadra of the infinite 1 -dimensional 
simplicial complexes $K(R)$ and $K\left(R_{+}\right)$, respectively, where

$$
\begin{gathered}
K(R)=\{n,[n, n+1]: n \text { is an integer }\} \\
K\left(R_{+}\right)=\{n,[n, n+1]: n \text { is a nonnegative integer }\}
\end{gathered}
$$

We have the following theorem.

Theorem 4.2. There exists a totally chaotic continuous map $f: R \rightarrow R$, which satisfies that $f\left(R_{+}\right) \subset R_{+}$, and $f \mid R_{+}$is also a totally chaotic map.

Proof. Let $c_{0}, c_{1}, c_{2}, \cdots$ be a given infinite sequence of positive numbers with $c_{k} \leq$ $1 / 2$ for every even number $k \geq 0$. We first choose a $C^{\infty}$ map $f_{0}: R \rightarrow R$ such that

(0.1) $f_{0}(0)=1, f_{0}(x)>x$ for any $x \leq 0$, and $\lim _{x \rightarrow-\infty} f_{0}(x)=-\infty$;

$(0.2) \quad f_{0}^{\prime}(x) \equiv 1-c_{0}$ for any $x \geq 1$ and $f_{0}^{\prime}(x)>0$ for all $x \in R$, where $f_{0}^{\prime}(x)$ is the derivative of $f_{0}(x)$;

(0.3) $1-c_{0}<f_{0}^{\prime}(x)<1$ for $0<x<1$.

The equation $f_{0}(x)=x$ has a unique root. Suppose this root is $a_{0}$. Then $a_{0}>2$. Obviously, there is an integer $n_{0} \geq 2$ such that $f_{0}^{n_{0}}([-1,1]) \subset\left[a_{0}-1, a_{0}\right)$. Let $\varepsilon_{0}=a_{0}-f_{0}^{n_{0}}(1)$. Then $0<\varepsilon_{0}<1$.

Next, we take a $C^{\infty}$ map $f_{1}: R \rightarrow R$ such that

(1.1) $f_{1}(x)=f_{0}(x)$ for any $x \leq a_{0}-\varepsilon_{0}$, and $f_{1}(x)>x$ for all $x \in R$;

(1.2) the derivative $f_{1}^{\prime}(x) \equiv 1+c_{1}$ for any $x \geq a_{0}$;

(1.3) $f_{0}^{\prime}(x)<f_{1}^{\prime}(x)<1+c_{1}$ for $a_{0}-\varepsilon_{0}<x<a_{0}$.

Evidently, there is an integer $n_{1}>n_{0}$ such that $f_{1}^{n_{1}}\left(-a_{0}\right)>2 a_{0}$, and the derivative $d\left(f_{1}^{n_{1}}(x)\right) / d x>2$ for all $x \in\left[-a_{0}, a_{0}\right]$. Let $a_{1}=f_{1}^{n_{1}}\left(a_{0}\right)$. Then $a_{1}>2 a_{0}$. Put $\varepsilon_{1}=\varepsilon_{0}$.

Now we choose again a $C^{\infty}$ map $f_{2}: R \rightarrow R$ such that

(2.1) $\quad f_{2}(x)=f_{1}(x)$ for any $x \leq a_{1}+1$;

(2.2) $f_{2}^{\prime}(x) \equiv 1-c_{2}$ for any $x \geq a_{1}+2$;

(2.3) $1-c_{2}<f_{2}^{\prime}(x)<1+c_{1}$ for $a_{1}+1<x<a_{1}+2$.

It is easy to see that the equation $f_{2}(x)=x$ has a unique root. Suppose this root is $a_{2}$. Then $a_{2}>a_{1}+1$. Clearly, there is an integer $n_{2}>n_{1}$ such that $f_{2}^{n_{2}}\left(\left[-a_{1}, a_{1}\right]\right) \subset\left[a_{2}-2^{-2}, a_{2}\right)$. Let $\varepsilon_{2}=a_{2}-f_{2}^{n_{2}}\left(a_{1}\right)$. Then $0<\varepsilon_{2}<2^{-2}$.

Continuing this process, for every positive integer $k=1,2,3, \cdots$, we can choose a $C^{\infty}$ map $f_{k}: R \rightarrow R$ and take a positive integer $n_{k}$ and two positive numbers $a_{k}, \varepsilon_{k}$ satisfying the following conditions:

(a) If $k \geq 1$ is odd, then

(k.1.a) $f_{k}(x)=f_{k-1}(x)$ for all $x \leq a_{k-1}-\varepsilon_{k-1}$, and $f_{k-1}(x)>x$ for all $x \in R$;

(k.2.a) the derivative $f_{k}^{\prime}(x) \equiv 1+c_{k}$ for all $x \geq a_{k-1}$;

(k.3.a) $f_{k-1}^{\prime}(x)<f_{k}^{\prime}(x)<1+c_{k}$ for $a_{k-1}-\varepsilon_{k-1}<x<a_{k-1}$;

(k.4.a) $n_{k}>n_{k-1}, f_{k}^{n_{k}}\left(-a_{k-1}\right)>2 a_{k-1}$, and the derivative $d\left(f_{k}^{n_{k}}(x)\right) / d x>2^{k}$ for all $x \in\left[-a_{k-1}, a_{k-1}\right]$;

(k.5.a) $\quad a_{k}=f_{k}^{n_{k}}\left(a_{k-1}\right)>2 a_{k-1}$, and $\varepsilon_{k}=\varepsilon_{k-1}$.

(b) If $k \geq 2$ is even, then

(k.1.b) $f_{k}(x)=f_{k-1}(x)$ for all $x \leq a_{k-1}+1$;

(k.2.b) $f_{k}^{\prime}(x) \equiv 1-c_{k}$ for all $x \geq a_{k-1}+2$;

(k.3.b) $1-c_{k}<f_{k}^{\prime}(x)<1+c_{k-1}$ for $a_{k-1}+1<x<a_{k-1}+2$;

(k.4.b) the equation $f_{k}(x)=x$ has a unique root, and $a_{k}$ is this root, $a_{k}>$ $a_{k-1}+1$;

(k.5.b) $n_{k}>n_{k-1}$, and $f_{k}^{n_{k}}\left(\left[-a_{k-1}, a_{k-1}\right]\right) \subset\left[a_{k}-2^{-k}, a_{k}\right)$; 
(k.6.b) $\quad \varepsilon_{k}=a_{k}-f_{k}^{n_{k}}\left(a_{k-1}\right) \in\left(0,2^{-k}\right]$.

From these conditions we know that there exists a limit function $f=\lim _{k \rightarrow \infty} f_{k}$ with $f(x)=f_{k}(x)$ for $x \leq a_{k}-\varepsilon_{k}$. Thus $f: R \rightarrow R$ is a $C^{\infty}$ map. For any given $u, v \in R$ with $u \neq v$, take a positive integer $j \geq 1$ such that $\{u, v\} \subset\left[-a_{j}, a_{j}\right]$. Then by (k.4.a) we have $\left|f^{n_{k}}(u)-f^{n_{k}}(v)\right|>2^{k}|u-v|$ for every odd $k>j$, and by (k.5.b) we have $\left|f^{n_{k}}(u)-f^{n_{k}}(v)\right|<2^{-k}$ for every even $k>j$. This implies that

$$
\liminf _{k \rightarrow \infty}\left|f^{k}(u)-f^{k}(v)\right|=0, \quad \text { and } \limsup _{k \rightarrow \infty}\left|f^{k}(u)-f^{k}(v)\right|=\infty
$$

Hence $f$ is totally chaotic.

Noting that $f(x)>x$ for any $x \in R$, we have $f\left(R_{+}\right) \subset R_{+}$. Therefore, $f \mid R_{+}$is also totally chaotic. Theorem 4.2 is proven.

Remark 4.3. Let $f: R \rightarrow R$ be as in the proof of Theorem 4.2. Then $f$ is a $C^{\infty}$ diffeomorphism. For any $n \geq 2$, define $F_{n}: R^{n} \rightarrow R^{n}$ by

$F_{n}\left(x_{1}, x_{2}, \cdots, x_{n}\right)=\left(f\left(x_{1}\right), f\left(x_{2}\right), \cdots, f\left(x_{n}\right)\right)$, for any $\left(x_{1}, x_{2}, \cdots, x_{n}\right) \in R^{n}$.

It is easy to see that $F_{n}$ is also a $C^{\infty}$ diffeomorphism, and $F_{n}$ is totally chaotic.

\section{REFERENCES}

[1] Ll. Alseda, J. Llibre and M. Misiurewicz, Periodic orbits of maps of $Y$, Trans. Amer. Math. Soc. 313(1989), 475-538. MR 90c:58145

[2] Ll. Alseda and J. M. Moreno, Linear orderings and the full periodicity kernel for the n-star, J. Math. Anal. Appl. 180(1993), 599-616. MR 95e:58141

[3] Ll. Alseda and X. D. Ye, No division and the set of periods for tree maps, Ergod. Th. \& Dynam. Sys. 15(1995), 221-237. MR 96d:58109

[4] M. A. Armstrong, Basic Topology, Springer-Verlag, New York, 1983. MR 84f:55001

[5] A. Bruckner and T. Hu, On scrambled set and chaotic functions, Trans. Amer. Math. Soc. 301(1987), 289-297. MR 88f:26003

[6] K. Jankova and J. Smital, A characterization of chaos, Bull. Austral. Math. Soc. 34(1986), 283-292. MR 87k:58178

[7] V. Jimenez, Large chaos in smooth functions of zero topological entropy, Bull. Austral. Math. Soc. 46(1992), 271-285. MR 93h:58099

[8] I. Kan, A chaotic function possessing a scrambled set of positive Lebesgue measure, Proc. Amer. Math. Soc. 92(1984), 45-49. MR 86b:26009a

[9] M. Kuchta and J. Smital, Two point scrambled set implies chaos, European Conference on Iteration Theory(ECIT 87), World Sci. Publishing Co., Singapore, 1989, pp.427-430. MR 91j:58112

[10] S. H. Li and X. D. Ye, Topological entropy for finite invariant subsets of $Y$, Trans. Amer. Math. Soc. 347(1995), 4651-4661. MR 96e:58052

[11] T. Y. Li and J. Yorke, Period three implies chaos, Amer. Math. Monthly 82(1975), 985-992. MR 52:5898

[12] V. J. Lopez, Paradoxical functions on the interval, Proc. Amer. Math. Soc. 120(1994), 465473. MR 94g:58141

[13] M. Misiurewicz, Chaos almost everywhere, Iteration Theory and its Functional Equations, Lecture Notes in Math., Vol.1163, Springer, Berlin, 1985, pp.125-130. MR 87e:58152

[14] Z. Nitecki, Differentiable Dynamics, The M.I.T. Press, Cambridge Mass., 1971. MR 58:31210

[15] J. Smital, A chaotic function with a scrambled set of positive Lebesgue measure, Proc. Amer. Math. Soc. 92(1984), 50-54. MR 86b:26009b

Institute of Mathematics, Shantou University, Shantou, Guangdong 515063, P. R. CHINA

E-mail address: jhmai@mailserv.stu.edu.cn 